\title{
Predicting growth of gold nanoparticle by photochemical reduction process associated with mathematical modelling
}

\author{
Hazidatul A. Hamlan ${ }^{1}$, Norma Alias ${ }^{2 *}$, Jia-Cherng Chong ${ }^{3}$, Hood-Hong Ley ${ }^{3}$, Noriah Bidin $^{3}$, Hamisan Rahmat ${ }^{1}$ \\ ${ }^{1}$ Department of Mathematic, Faculty of Science, Universiti Teknologi Malaysia, 81310 UTM Johor Bahru, Johor, Malaysia, \\ ${ }^{2}$ Center for Sustainable Nanomaterials, Ibnu Sina Institute for Scientific and Industrial Research, Universiti Teknologi Malaysia, 81310 UTM Johor \\ Bahru, Johor, \\ ${ }^{3}$ Department of Physic, Faculty of Science, Universiti Teknologi Malaysia, 81310 UTM Johor Bahru, Johor, Malaysia, \\ “Corresponding Author: norma@ibnusina.utm.my
}

\section{Article history :}

Received 2 November 2015

Accepted 15 November 2015

\section{GRAPHICAL ABSTRACT}

Studying the uniqueness of gold nanoparticles (AuNPs) through experimental processes

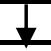

Govern the mathematical modeling for growth of AuNPs

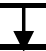

Solving the governing equation using numerical approaches

Investigate the numerical analysis

\begin{abstract}
Nowadays, to develop new generation of nanodevices, most researchers such as chemists, physicists, biologists, even the engineers are focusing their studies towards the uniqueness as well as the chemical properties of metal nanoparticles. Even, the mathematician also has governed the appropriate mathematical modelling regarding of properties of nanoparticles such as gold nanoparticles. In this paper, some experiment regarding the photochemical and photocatalytic processes for predicting the growth of gold nanoparticles from previous studies has been reviewed. Besides that, in observing the growth rate of gold nanoparticles, a mathematical modelling has been governed. Where, ultraviolet, UV radiation with wavelength of $366 \mathrm{~nm}$ and $253.7 \mathrm{~nm}$ has been fixed as the constant parameters. The governing equation is then solved numerically using some iterative method known as Jacobi and Gauss Seidel. The convergence of both methods is discussed in detail and the numerical analysis is presented in table form to justify and validate the convergence as well as the performance of the proposed iterative methods.

Keywords: photochemical, parabolic PDE, gold nanoparticles, mathematical model, Finite Different Method (FDM)

(C) 2015 Penerbit UTM Press. All rights reserved http://dx.doi.org/10.11113/mjfas.v11n3.386
\end{abstract}

\section{INTRODUCTION}

Nowadays, there are various experiment has been conducted regarding the issues of gold nanoparticle (NPs) based on ultraviolet radiation. As an example, Sarina et al. (2013) has investigated the performance of photocatalysts through the properties of (NPs) using light irradiation [1]. While, in 2010 , by using a photochemical initiator known as (Irgacure-2959), Hamaguchi et al. has employed the photochemical synthesis of glycine-stabilized gold nanoparticles (Au NPs) at specific room temperature which is $27^{\circ} \mathrm{C}$ towards heavy metals [2]. Size- and shapedependent properties of NPs, such as optical, electronic, and catalytic properties have a very high significance. Thus, these properties have been studied extensively with the aim of controlling the size and shape of the NPs $[3,4]$. Both photochemical and photocatalysts process has been widely investigated by chemists and physicists in order to value the properties of gold nanoparticles.

In controlling the size and shape of Au NPs, both researchers mention before has employed photochemical process with the presence of ultraviolet irradiation. Generally, photochemical term is employed for describing the reaction of some chemical caused by presence and immersion of ultraviolet with wavelength from 100 to 400 $\mathrm{nm}$, visible light with wavelength of 400 to $750 \mathrm{~nm}$ as well as infrared radiation $(750-2500 \mathrm{~nm})$ [5]. Photochemical process is begun by the atom or molecule absorbing a quantum of light energy from a photon. The purpose of absorbing the light energy is to increases the atom or molecule above its normal level and photochemical reaction is also known as photolysis [6].

Due to short review above, this paper is proposed for visualizing the growth of NPs, a photochemical reduction process using ultraviolet radiation with wavelength of $366 \mathrm{~nm}$ and $253.7 \mathrm{~nm}$. The method used is by modifying Turkevich pathway of fabricating water suspended with gold nanoparticles. Besides that, to validate the experiment, some mathematical modelling has been proposed in order to visualize the growth of gold nanoparticle. The simulation of mathematical model has been obtained using some numerical approaches. 


\section{EXPERIMENTS}

Chemicals substance used for conducting this photochemical process for gold nanoparticles were purchased from Sigma Aldrich. Before begins the experiment, all apparatus involved in the processes was prewashed using aqua regia. While, generous amount of ultrapure deionised water (18 M $\Omega \mathrm{SG}$ systems) is used for rinsing all the glassware.

The purpose of prewashed the apparatus used in this process is for removing the possible nucleation sites. This photochemical process is started by providing a stock solution of $1.5 \mathrm{mM}$ auric acid $\left(\mathrm{HAuCl}_{4} \cdot 3 \mathrm{H}_{2} \mathrm{O}\right)$ and $1 \% \mathrm{w} / \mathrm{v}$ tri-sodium citrate $\left(\mathrm{Na}_{3} \mathrm{C}_{6} \mathrm{H}_{5} \mathrm{O}_{7} .2 \mathrm{H}_{2} \mathrm{O}\right)$ separately. Besides that, by following the protocol that listed in Table 1 below, a set of AuNP samples was synthesized by adding trisodium citrate into the auric acid. In this experiment, two types of ultraviolet light sources were used with UVA radiation from 4 watts Wood's fluorescent lamp and UVC emissions from quartz enclosed mercury discharge tube having singular peak of $366 \mathrm{~nm}$ and principal peak at 253.7 $\mathrm{nm}$ respectively. All of chemical samples are photochemically initiated and they were exposed through an aperture of $1 \mathrm{~cm}^{2}$ at irradiance of $0.8 \mathrm{~mW} \mathrm{~cm}$. The UVC irradiated samples is controlled and monitored by completing the filtering of shortwave bands with $0.15 \mathrm{~mm}$ thick borosilicate glass.

The exposure time for sample A was recorded from injecting tri-sodium citrate into rigorously stirring boiling auric acid. Separate aliquots was extracted as the colour change and cooled rapidly in ice bath to stop further agglomeration process. The absorption spectrum of the aliquot was investigated immediately by a fibre spectrophotometer set-up (Avantes CUV-ALL-UV/VIS) with Ocean Optics USB4000 and a tungsten-halogen light source. The final optical absorbance is recorded for sample A when its colour reached a brilliant ruby red. After synthesis, acidity of all the samples was measured periodically by a digital $\mathrm{pH}$ meter and they were kept for 8 days. The size distributions of the samples which are gold nanoparticles (Au NPs) were measured by photon correlation spectroscopy with Malvern Zetasizer Nano ZS.

\section{MATHEMATICAL MODEL}

From a decade ago, many applications have been modelled using parabolic equation. As an example, Alias et al. has studied multidimensional parabolic equations of PDE as a tool to visualize brain tumor growth [7], while
Castor \& Sturm (2008) have performed the simulation on underwater acoustic propagation with $3 \mathrm{D}$ parabolic equation [8]. Mathematical models of PDE usually present the analytical and numerical challenges in describing the behaviour of interacting agents. In solving the PDE, most researchers used complex systems that involving multi scales parameters to represent the real life problems. According to the literature reviews, the contribution of this paper is applying 1D parabolic partial differential equation (PDE) to obtain the growth of gold NPs.

This model is straight forward implemented on FDM, involved numerical simulation and potential to visualize the growth of gold NPs in 1D and 2D scalar fields. Referring to Equation (1), the initial condition equation to fix the existence of exact solutions in physicalchemistry experimental results under consideration is as below,

$\frac{\partial U}{\partial t}=\Phi \frac{\partial^{2} U}{\partial x^{2}}+\lambda, \quad 0 \leq x \leq 1, \quad 0<t$,

With initial condition,

$$
U(x, 0)=\sin (\pi x), \quad 0 \leq x \leq T,
$$

and the boundary condition are given as below,

$$
\begin{array}{ll}
U(0, t)=0, & 0 \leq x \leq 1, \\
U(1, t)=0, & 0 \leq x \leq 1,
\end{array}
$$

where, $\lambda$ represent the incoherent ultraviolet radiation, $\Phi$ is diameter of gold growth, $U$ is the rate of gold growth, $t$ is time (duration taken for the growth rate) and $\mathrm{x}$ is spatial coordinate in $\mathrm{x}$ direction. In solving the mathematical model above, FDM is straight forward employed.

\subsection{Finite Different Method (FDM)}

In converting the governing equation (1) into simplest form which is Linear System Equation (LSE), the discretization is needed. Thus, in this study, Finite Different Method (FDM) is employed for discretizing purpose associated to the mesh size of NPs, motion of physical parameter properties, particular form of the growth, and free surface of the targeted region. Compare to Finite Element Method (FEM) and Finite Volume Method FVM, FDM is simplest ways, to gain NPs meshes of data points [9]. The discretization using FDM approach is depends on the dimension of the region, the order as well as the types of derivative. By applying these techniques, the discretization of Equation (1) is given by Equation (1.a),

\begin{tabular}{|c|c|c|c|c|c|}
\hline Sample & $\begin{array}{l}\mathrm{HAuCl}_{4} \\
\text { Vol. [ml] }\end{array}$ & $\begin{array}{c}\text { Na-citrate } \\
\text { Vol. }[\mu l]\end{array}$ & $\begin{array}{l}\text { Au : Citrate } \\
\text { ionic ratio }\end{array}$ & Synthesis method & $\begin{array}{c}\text { Exposure time } \\
\text { [minutes] }\end{array}$ \\
\hline $\mathbf{A}$ & 30 & 130 & 2.036 & UVC photo-initiation & 5 \\
\hline B & 30 & 150 & 1.764 & UVA photo-initiation & 10 \\
\hline $\mathbf{C}$ & 30 & 150 & 1.764 & UVC photo-initiation & 10 \\
\hline D & 30 & 120 & 2.206 & UVA photo-initiation & 15 \\
\hline $\mathbf{E}$ & 30 & 120 & 2.206 & UVC photo-initiation & 15 \\
\hline $\mathbf{F}$ & 30 & 300 & 0.882 & UVC photo-initiation & 10 \\
\hline
\end{tabular}
where $0 \leq \theta \leq \frac{1}{2}$ and $\frac{1}{2} \leq \theta \leq 1$

Table 1 Synthesis protocol for all samples involves 


$$
\begin{gathered}
\frac{U_{i}^{j+1}-U_{i}^{j}}{\Delta t}=\Phi( \\
\left.+\lambda\left(\delta_{x}^{2}\right) U_{i}^{j+1}+(1-\theta)\left(\delta_{x}^{2}\right) U_{i}^{j}\right) \\
+\lambda
\end{gathered}
$$

By applying the forward difference formula for first order derivative and 3 points discretization for second order derivative, the Equation (1.a) can be simplifies as below;

$$
\begin{aligned}
-r \theta U_{i-1}^{j+1}+(1+ & 2 r \theta) U_{i}^{j+1}-r \theta U_{i+1}^{j+1} \\
& =r(1-\theta) U_{i-1}^{j}+(1 \\
& -2 r(1-\theta)) U_{i}^{j} \\
& +r(1-\theta) U_{i+1}^{j}+\lambda \Delta t
\end{aligned}
$$

where, $r=\frac{\Phi \Delta t}{(\Delta x)^{2}}, i=1,2, \ldots, m$ and $j=1,2, \ldots, n$.. Besides that, the Linear System Equation (LSE) of standard scheme for the three point's discretization for one dimensional parabolic type of PDE in Equation (1.b) can be visualize in matrix form, such as;

$$
A U=f
$$

where $U$ and $f$ is one dimensional vector that define as below;

$$
\begin{aligned}
U & =\left(U_{1, j+1}, U_{2, j+1}, U_{3, j+1}, \ldots, U_{m, j+1}\right)^{T} \\
f & =\left(f_{1}, f_{2}, f_{3}, \ldots, f_{m}\right)
\end{aligned}
$$

Equation (1.c) above can be write in the matrix form as below;

$$
\left[\begin{array}{cccccc}
a & b & & & & \\
c & a & b & & & 0 \\
& c & a & b & & \\
& & \ddots & \ddots & \ddots & \\
0 & & & c & a & b \\
& & & & c & a
\end{array}\right]_{(m \times m)}\left[\begin{array}{c}
U_{1} \\
U_{2} \\
U_{3} \\
\vdots \\
U_{m-1} \\
U_{m}
\end{array}\right]_{(m \times 1)}=\left[\begin{array}{c}
f_{1} \\
f_{2} \\
f_{3} \\
\vdots \\
f_{m-1} \\
f_{m}
\end{array}\right]_{(m \times 1)}
$$

where,

and,

$$
a=1+2 r \theta, \quad b=c=-r \theta
$$

$$
\begin{gathered}
f_{1}=r(1-\theta) U_{0}^{j}+(1-2 r(1-\theta)) U_{1}^{j}+r(1-\theta) U_{2}^{j}+r \theta U_{0}^{j+1} \\
+\lambda \Delta t \\
\begin{aligned}
& f_{i}=r(1-\theta) U_{i-1}^{j}+(1-2 r(1-\theta)) U_{i}^{j}+r(1-\theta) U_{i+1}^{j}+\lambda \Delta t, \\
& \text { for } i=2,3, \ldots . m-1 \\
& f_{m}=r(1-\theta) U_{m-1}^{j}+(1-2 r(1-\theta)) U_{m}^{j}+r(1-\theta) U_{m+1}^{j}+ \\
& r \theta U_{m+1}^{j+1}+\lambda \Delta t .
\end{aligned}
\end{gathered}
$$

\subsection{Iterative Scheme and Algorithm}

The LSE that obtained from the discretization in Section 3.1 above is then solved with some iterative numerical methods. The methods that are being highlighted in this paper are known as Jacobi (JB) and Gauss Seidel methods.

\section{A)}

Jacobi Method (JB)

The step sizes of nanoscale gold NPs growth during photochemical reduction process can be measure using Jacobi method approach. As we already concern this method is essential for solving LSE (1.d) by computing the step sizes of growth gold NPs with respected to time and space variables. Equation (2) below describe the JB computation.

$$
U_{i}^{(k+1)}=\frac{1}{a_{i i}}\left(c_{i}-\sum_{j \neq i} a_{i j} U_{j}^{(k)}\right), i=1,2, \ldots, n
$$

The governing equation is convergent with JB method and able to express the growth of particle at a new time step depends on the few forms of points at the previous time. This method is uniquely designed in the closed domain and available to generate a large sparse fine domain for high resolution of growth visualization. The iteration for this method is repeated until it reaches the stopping criterion such that $\left|U_{i}^{(k+1)}-U_{i}^{(k)}\right| \leq \varepsilon$ where $\varepsilon$ is set as $1.0 \times 10^{-4}$.

\section{B) Gauss Seidel Method (GS)}

Second iterative method that used for solving the LSE in section 3.1 is Gauss Seidel (GS) Method. This method is modification (improvement) from Jacobi method, so it is no more difficult to apply and it is often requires fewer iteration to produce the same degree of accuracy.

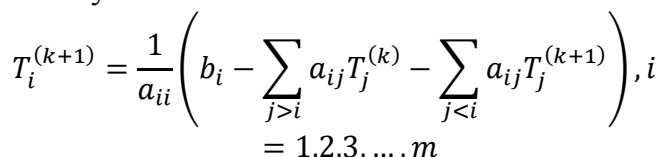

Gauss Seidel method computes the calculation by employing the updated value of $U_{i}$ and the computation of predicting the growth of gold nanoparticles using GS method can be simplifies as Equation (3).

Besides that, in predicting the growth of gold nanoparticles, an algorithm has been developed based on the iterative method above. The algorithm involve in this research is known as sequential algorithm and can be obtained from [10-12]. The computation for this algorithm is begun by simulating the initial and boundary conditions, followed by computation of the numerical iterative methods which are known as Jacobi and Gauss Seidel method.

\section{RESULT AND DISCUSSION}

In this section, the experimental result as well as the simulation results from governing equation of parabolic PDE is presented. Since the simulation is been done using numerical approach, thus the numerical analysis also being discussed detailed at the end of this section

\subsection{Experimental Result}

Through the photochemical synthesis of AuNPs, tri-sodium citrate is added into boiling gold chloride dilution and has produced relatively monodisperse AuNPs with diameter between 10 to $20 \mathrm{~nm}$ [11]. The changes of solution appearance during the experiment were monitored in order to indicate the presence of AuNPs in each sample as shown in Figure 1 below. 


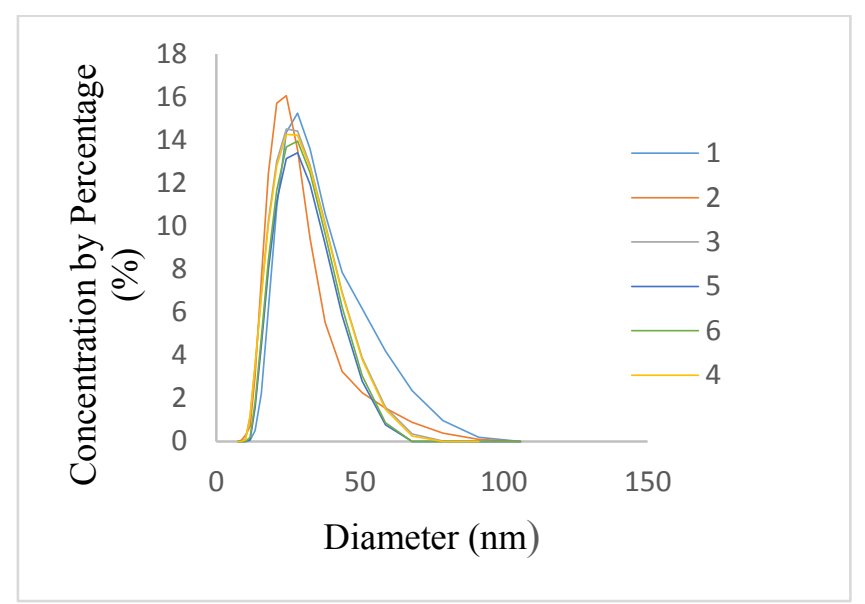

Fig. 1 Particle size distribution of sample $A=1, B=2$, $\mathrm{C}=3, \mathrm{D}=5, \mathrm{E}=6, \mathrm{~F}=4$ (synthesis using $\mathrm{UV}$ )

Figure 2 shows the absorption spectra that were measured using photon correlation spectroscopy for Sample A and D. Refer to Table 1, Sample A and D were synthesized using different synthesis methods, which are UVA and UVC photo-initiation. Based on Figure 2, it is clearly shown that, UVC initiated samples tend to exhibit faster and narrower compared to samples that initiated by UVA. This effect might be contributed by water photolysis which occurs with exposure to UVC rather than UVA wavelengths.

\subsection{Simulation of Governing Equation}

Different diameters are used in order to visualize the rate of growth for gold NPs. As shown in Figure 3, average particle size (diameter) from 5 to $100 \mathrm{~nm}$ depending on ultraviolet wavelength is used. In this study, six different sizes of AuNPs are measured, which are 5, 20, 40, 60, 80 and $100 \mathrm{~nm}$. From Figure 3, it is conclude that, the biggest size of diameter, $\Phi$ used, give the highest rate of gold growth, $U(x, t)$.

By focusing on the highest rate of gold growth, with diameter $100 \mathrm{~nm}$, the simulation of mathematical model from Equation (1.b) are charged by different amounts of UV radiation with wavelength of $366 \mathrm{~nm}$ and $253.7 \mathrm{~nm}$. The visualization graph from governing the equation is described by Figure 4 below.

Besides that, numerical analysis in terms of run time, number of iterations, root mean square error (RMSE) as well as the computational complexity is discussed detailed in this section. Matrix size and grid values for $\mathrm{x}$ and t-axes used to simulate the algorithm are stated in Table 2. The simulation for the algorithm will be stopped as it satisfies the stopping criterion, which is $1.0 \times 10^{-4}$.
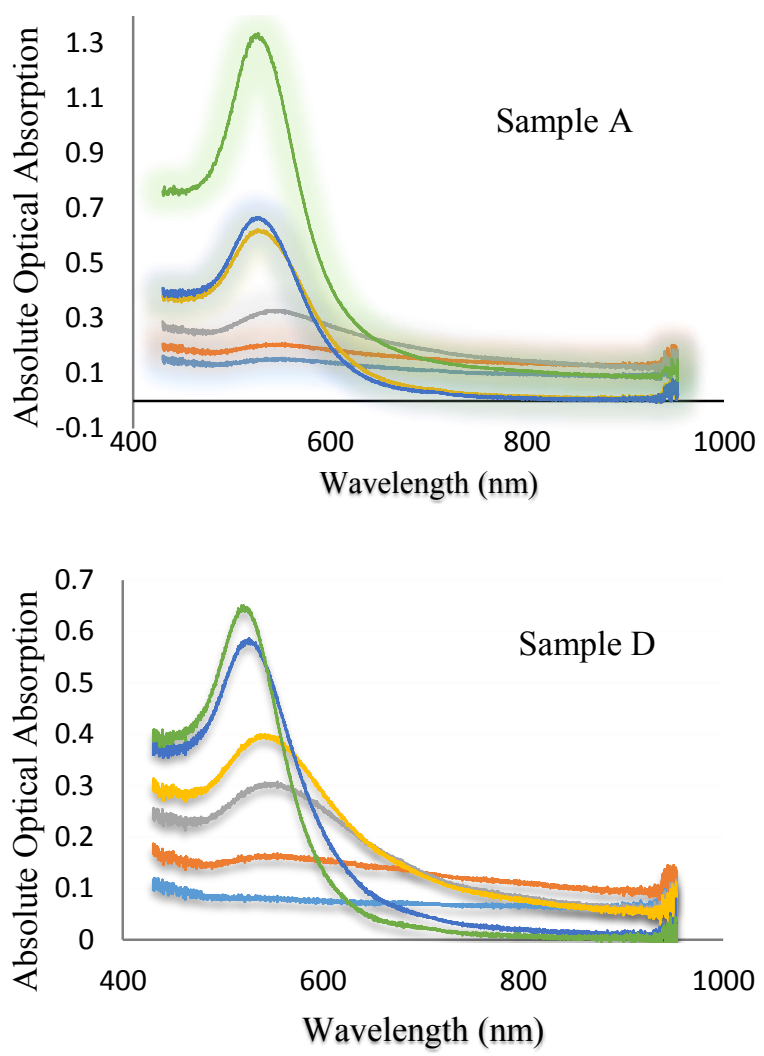

Fig. 2 Corresponds absolute optical absorption against wavelength in nanometers for Sample A and D

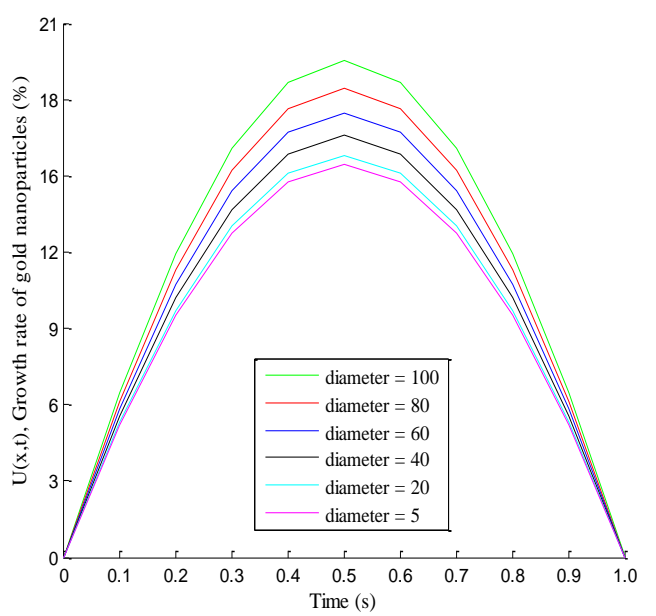

Fig. 3 Visualization of growth for gold NPs with different values of diameters

Run time of a given task can be defined as the time that spent by the system in executing task and best known as execution time. Time execution depends on the computational complexity value [10]. As observed from Table 2 below, Jacobi method takes 4.048439s in order to complete the computation of algorithm that stated in Figure 4. Compare to Gauss Seidel method, the performance in term of execution time for Jacobi method is lower. Since, using Gauss Seidel method, the computation of the 
algorithm has completed within 2.813832 s. The execution time is depended on the computational complexity mathematical model from PDE. Since the Jacobi method have huge calculation for both addition and multiplication, thus its record higher computation time compare to Jacobi.

The computational and complexity of a mathematical model from PDE can be measured by determine the total of addition and subtraction operation involve as well as its multiplication and division. The number of steps is measured as a function of that matrix size. Variable $\mathrm{m}$ represents the matrix size of the algorithm.

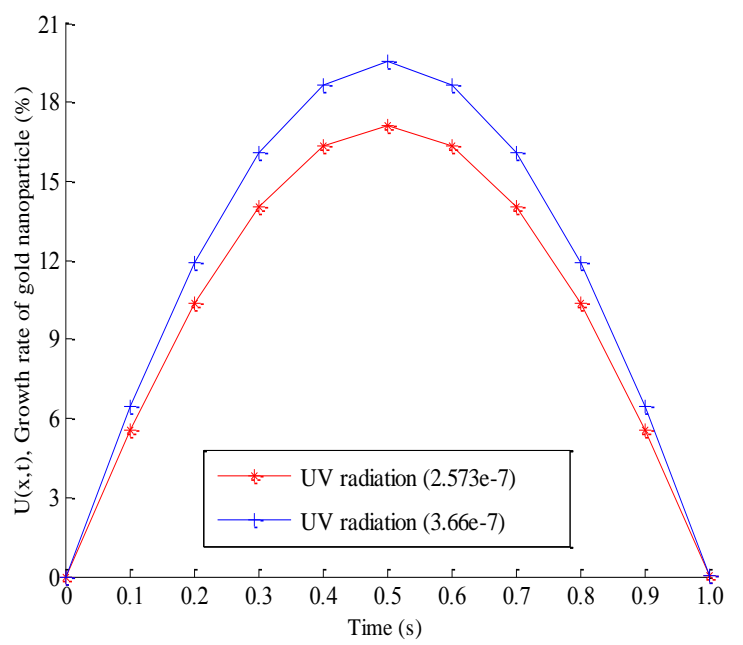

Fig. 4 Visualization of growth for gold NPs with different UV light radiation wavelengths ( 366 and $253.7 \mathrm{~nm}$ )

Table 2 Numerical Analysis of the Iterative Methods

\begin{tabular}{|c|c|c|}
\hline \multirow[t]{2}{*}{ Numerical analysis } & \multicolumn{2}{|c|}{ Schemes } \\
\hline & Jacobi & Gauss Seidel \\
\hline$\Delta x, \Delta t$ & 0.01 & 0.01 \\
\hline Matrix sizes, $m$ & 100 & 100 \\
\hline Tolerance, $\varepsilon$ & $1.0 \times 10^{-4}$ & $1.0 \times 10^{-4}$ \\
\hline Run time (sec) & 4.048439 & 2.813832 \\
\hline Iterations number & 125 & 70 \\
\hline RMSE & $\begin{array}{l}1.683945651 \\
\times 10^{-4}\end{array}$ & $\begin{array}{l}1.4305853381 \\
\times 10^{-4}\end{array}$ \\
\hline Comp. & 750 & 696 \\
\hline Complexity $(\times / \div)$ & 625 & 609 \\
\hline
\end{tabular}

\section{CONCLUSION}

The main contribution of this paper is being able to visualize and model the growth of gold nanoparticles in one-dimensional space. We had identified the onedimensional parabolic equation described the growth of gold nanoparticles based on the rate of growth, in time, $t$ and space, $x$ as the physical state of the system. The parabolic equations is derived using numerical FDM and a weighted approximations equations has been formed to obtain the values of the rate growth for gold nanoparticles $U_{i, k}$.

The Linear System Equation (LSE) that is obtained from discretization of the governing equation is solved using some iterative numerical methods known as Jacobi and Gauss Seidel methods. In order to validate the numerical scheme, the obtained data were compared with experimental result using photochemical process in detecting the growth of gold nanoparticle. The visualization of the mathematical modeling using MATLAB software has shown that the rate of gold growth as opposed to time.

The results obtained have proved that the mathematical model is capable to simulate the rate of gold growth through numerical scheme approach. The implementation of Jacobi method is much simpler than Gauss Seidel method. However, result obtained by Gauss Seidel method shows reasonably good agreement and more accurate rather than Jacobi method. This is due to their numerical analysis that stated in Table 2 above, where the iteration number as well as the execution time to solve the computation using Gauss Seidel method is faster that Jacobi method. The mathematical model used for determine the rate of gold NP growth can be improve with various parameters, for future research.

\section{ACKNOWLEDGEMENTS}

This research is supported in part by the Ministry of Higher Education (MOHE), Research Management Centre (RMC) and University Technology Malaysia (UTM) through Research University Grant (09H71) and the authors are grateful to Ibnu Sina Institute, University Technology Malaysia for the excellent support to this research.

\section{REFERENCES}

[1] S. Sarina, E.R. Waclawik, H. Zhu, Green Chem. 15 (2013) 1814.

[2] K. Hamaguchi, H. Kawasaki, R. Arakawa, Colloid Surf. A 367 (2010) 167.

[3] A.R. Tao, S. Habas, P. Yang, Small 4 (2008) 310.

[4] Y. Xia, Y. Xiong, B. Lim, S. E. Skrabalak, Angew. Chem. Int. Ed. 48 (2009) 60

[5] A.D. McNaught, A. Wilkinson, IUPAC. Compendium of Chemical Terminology, 2nd ed. (the "Gold Book"). 1997.

[6] Z.C. Kornblum, Photochemistry Encyclopedia Americana. Retrieved July 24, 2010, from Grolier Online http://ea.grolier.com/article?id=0311750-00

[7] N. Alias, M. Masseri, I. Safa, M. Islam, S.N. Khalid, J. Appl. Sci. 9 (2009) 505.

[8] K. Castor, F. Sturm, J. Comput. Acoust. 16 (2008) 137.

[9] H. Huang, Polymer and tissue separation and micro/nanofabrication via ultra-short pulsed laser plasma-mediated ablation. Rutgers University-Graduate School-New Brunswick, 2010.

[10] Z.S.A. Ghaffar, N. Alias, F.S. Ismail, A.H.M. Murid, H. Hassan, Malay. J. Fund. Appl. Sci. 4 (2009) 379.

[11] J. Turkevich, P.C. Stevenson, J. Hillier, Discuss. Faraday Soc. 11 (1951) 55 .

[12] N. Alias, M. R. Islam, T. Ahmad, M. A. Razzaque. Fourth International Conference and Workshops on Basic and Applied Sciences ( $4^{\text {th }}$ ICOWOBAS) and Regional Annual Fundamental Science Symposium 2013 (11 ${ }^{\text {th }}$ RAFSS) (2013) 1-6. 